\title{
Synergistic effects of arsenic trioxide combined with Salmonella typhimurium in treating the advanced hepatocellular carcinoma in rat models
}

\author{
Qing Zhao, Ying Wang, Wen-Tao Li \\ Department of Interventional Radiology, Fudan University Shanghai Cancer Center, Shanghai, China \\ Contributions: (I) Conception and design: WT Li; (II) Administrative support: WT Li; (III) Provision of study materials or patients: Q Zhao; (IV) \\ Collection and assembly of data: Q Zhao, Y Wang; (V) Data analysis and interpretation: Q Zhao; (VI) Manuscript writing: All authors; (VII) Final \\ approval of manuscript: All authors. \\ Correspondence to: Wen-Tao Li, PhD. Department of Interventional Radiology, Fudan University Shanghai Cancer Center, No.270 Dongan Road, \\ Shanghai 200032, China. Email: shcc3844@163.com.
}

Background: To evaluate the safety and efficacy of arsenic trioxide (ATO) combined with VNP20009 in treating the advanced hepatocellular carcinoma (HCC).

Methods: The proliferation assay, migration assay and real-time PCR analyses were performed to assess the impact of ATO combined with VNP20009 on the McA-RH7777 cells. Forty Buffalo rats were orthotopically implanted with HCC in the livers and randomly divided into four groups: (A) ATO plus VNP20009; (B) ATO; (C) VNP20009; and (D) control. ATO (2 mg/kg) was administered by peritoneal injection once a day and continued for five days. VNP20009 (about $1 \times 10^{7} \mathrm{CFU}$ ) was directly injected into the tail vein. MRI examinations were performed to access the tumor responses one and 2 weeks later, respectively. Micro CT scans of chest were performed to assess the lung metastases. Hematoxylin-eosin (HE) staining and immunohistochemical analyses were performed to analyze the tumor tissues.

Results: In the in vitro experiments, VNP20009 suppressed the proliferation of McA-RH7777 cells, attenuated their migration ability, and weakened the potential of metastases. MRI examinations showed that the mean residual tumor volumes of ATO plus VNP20009 group on the 7th day and 14th day after the administration of ATO combined with VNP20009 were significantly smaller than those of other groups. Micro CT scans revealed that the lung metastases rates of ATO plus VNP20009 group and VNP20009 group were significantly lower than those of other groups. Immunohistochemical analyses displayed that the levels of VEGF and Vimentin in the tumors of ATO plus VNP20009 group were obviously lower than those of other groups. The median survival of rats in the ATO plus VNP20009 group was longer than those of other groups.

Conclusions: The strategy of ATO combined with VNP20009 was safe and had a potential to inhibit tumor growth, decrease the lung metastases, and prolong the overall survival in treating the advanced HCC. The two complementary interventions may have synergistic effects.

Keywords: Anaerobic bacteria; arsenic trioxide (ATO); hepatocellular carcinoma (HCC); Salmonella typhimurium

Submitted Jan 10, 2021. Accepted for publication Jun 08, 2021.

doi: 10.21037/jgo-21-4

View this article at: https://dx.doi.org/10.21037/jgo-21-4 


\section{Introduction}

Hepatocellular carcinoma (HCC) is the most common primary liver cancer. Worldwide, liver cancer ranks sixth in terms of incident cases and is one of the most lethal tumors (1). Arsenic trioxide (ATO) is an ingredient of the traditional Chinese medicinal arsenic, which has shown satisfactory efficacy in treating myelogenous leukemia (2). In recent years, it was reported that ATO might serve as an effective agent in the therapy of HCC $(3,4)$. However, since the agent is transported to tumor issues through blood flow, its anti-tumor efficacy may be limited within the poorly vascularized compartments of tumors. Besides, the hypoxia due to ischemia may promote the resistance of ATO in the advanced HCC (5). Therefore, complementary approaches are required to improve the efficacy of ATO in treating the advanced HCC.

Anaerobic bacterium has the potential to treat the hypoxic area of tumors. More than 100 years ago, physicians found that tumors regressed in some cancer patients suffering from the bacterial infection $(6,7)$. Since then, various bacterial strains had been investigated as anti-tumor agents (8). In the past few years, much attention had been attracted on cancer treatment using the anaerobic bacteria of Salmonella typhimurium (9-11). VNP20009, which is a genetically modified strain of Salmonella typhimurium with attenuated virulence and reduced risk of septic shock, is a kind of facultative anaerobia. As a kind of tumor-targeting anaerobia, VNP20009 is capable of colonizing the hypoxic tumor tissues and exerting adverse influence on the tumor microenvironment. VNP20009 had been investigated as a safe and effective anticancer agent in quite a few animal experiments and clinical trials $(9,12)$. Therefore, the mechanisms of ATO and VNP20009 in treating tumor may be complementary.

To our knowledge, there are no previous studies that have investigated the strategy of ATO combined with VNP20009 in treating cancers. This study aimed to evaluate the safety and efficacy of ATO combined with VNP20009 in treating the advanced HCC in rat models. We present this study in accordance with the ARRIVE reporting checklist (available at https://dx.doi.org/10.21037/jgo-21-4).

\section{Methods}

Experiments were performed under a project license (No. FUSCC-IACUC-S20190337) granted by institutional ethics board of Fudan University Shanghai Cancer Centre, in compliance with Fudan University guidelines for the care and use of animals.

\section{Cell line, bacteria}

In our study, the cell line of McA-RH7777 was used, which was a kind of HCC of Buffalo rat. Its biological property was very similar with that of the human HCC (13). The cell line of McA-RH7777 was obtained from the American Type Culture Collection (no. CRL1601; ATCC, Manassas, VA, USA). Dulbecco's Modified Eagle's Medium (DMEM, Thermo Fisher Scientific, Waltham, MA, USA) including $1 \%$ streptomycin and penicillin as well as $10 \%$ fetal bovine serum was utilized to incubate the tumor cells. The cell line was cultured at $37{ }^{\circ} \mathrm{C}$ in $5 \% \mathrm{CO}_{2}$. The bacteria of VNP20009 (ATCC 202165, Manassas, USA) was prepared as previously reported (14).

\section{Cell proliferation assays}

CCK-8 kits were used to evaluate the influence of ATO combined with VNP20009 on cell proliferation according to the instructions. VNP20009 $\left(1 \times 10^{6}\right.$ per well $)$ and/or ATO $(2 \mathrm{mg} / \mathrm{L})$ were added to McA-RH7777 cells when the cell density was $1 \times 10^{4}$ per well in 96 -well plates. The cells were incubated in a humidified incubator set at $37^{\circ} \mathrm{C}$ and $5 \%$ $\mathrm{CO}_{2}$ for 72 hours.

\section{Migration assays}

Transwell chambers (Corning, USA) were used to assess the impact of VNP 20009 in combination with ATO on the McA-RH7777 cells. The cell counting was performed before and after the migration assays. McA-RH7777 cells were plated at a density of $1 \times 10^{4}$ cells per well in 96-well plates. VNP $20009\left(1 \times 10^{6}\right.$ per well) and ATO $(2 \mathrm{mg} / \mathrm{L})$ were added to the cells, which were incubated for 12 hours.

\section{Real-time PCR analyses}

McA-RH7777 cells were seeded at a density of $1 \times 10^{4}$ cells per well in 96-well plates. The cells were incubated with VNP $20009\left(1 \times 10^{6}\right.$ per well $)$ and/or ATO $(2 \mathrm{mg} / \mathrm{L})$ for 1 hour. After being extracted from the infected cells by the TRIzol reagent (Invitrogen, Carlsbad, USA), the RNAs were reverse transcribed into cDNA using a M-MLV qPCR Kit (Invitrogen, Carlsbad, USA). 


\section{Animal models}

All animal experiments were ethically approved by our institute. The Buffalo rats ( 8 weeks, male, $80-100 \mathrm{~g}$, Charles River Laboratories, Wilmington, USA) were selected for in vivo experiments in this study. All the operations were performed under anesthesia. The rats were anaesthetized by intravenous injection with $2 \%$ pentobarbital sodium at a dose of $30 \mathrm{mg} / \mathrm{kg}$. To obtain the solid tumor of HCC for establishing rat models, McA-RH7777 cells (about $1 \times 10^{6}$ ) were subcutaneously injected into the inguinal area of a Buffalo rat. Three weeks later, the tumor was excised and cut into fragments (about $1 \mathrm{~mm}^{3}$ ) when it grew to be as large as $1 \mathrm{~cm}$ in diameter. To establish the orthotopic HCC animal models, abdominal incisions were made along the upper midline of rats to expose the livers. Then, the tumor fragments were implanted in the left lobes of livers under the scope of a magnification microscope (Nikon, Tokyo, Japan) (15). After sewing up the incisions, animals were sent back to the cages and had free access to the diet.

\section{Bacterial infection and ATO administration}

Three weeks after the orthotopic implantation of HCC fragments, MRI (3.0 T, Magnetom avanto, Siemens, Germany) examinations were performed to evaluate the tumor growth, as MRI owned higher per-lesion sensitivity than multidetector CT and was supposed as the preferred imaging modality for the diagnosis of HCC (16). Forty rats with liver tumors measuring about $1 \mathrm{~cm}$ in diameter were selected for the following experiments. The animals were randomly divided into four groups (10 rats each group): (A) ATO plus VNP20009; (B) ATO; (C) VNP20009; and (D) control. The bacteria of VNP20009 (about $1 \times 10^{7} \mathrm{CFU} / \mathrm{mL}$ ) was directly injected into the tail veins of rats in the (A) and (C) groups. ATO was administered $(2 \mathrm{mg} / \mathrm{kg} / \mathrm{day}$, once a day for 5 days) by the means of intraperitoneal injection for the (A) and (B) groups. The dose of ATO in this study was chosen according to the instructions of clinically available ATO products. The control group received administration of equivalent phosphate buffered saline (PBS).

\section{Analyses of anti-tumor efficacy}

MRI were utilized to evaluate tumor responses on the 7 th day and 14th day after the administration of ATO and VNP20009, respectively. The following formula was used to calibrate the tumor volumes: (length $\times$ width $\left.^{2}\right) / 2(17)$. Since
CT was considered as the cornerstone of imaging studies in the evaluation of lung cancer (18), micro-CT scans of chest were performed to access the metastatic progression two weeks after the administration of ATO and VNP20009. Five rats of each group were sacrificed in the method of cervical dislocation after undergoing the micro-CT scans. Death of rats were confirmed by lack of heartbeat, respiratory arrest, and lack of reflex. The remaining animals were observed for survival research. When the weight of rat decreased by nearly $20 \%$ or the rat had serious difficulties in moving, eating, and drinking due to the tumor load with or without the bacterial infection, euthanasia was performed by cervical dislocation to reduce the suffering.

\section{Hematoxylin-eosin (HE) staining and immunobistochemical analysis}

After the rats were sacrificed, tumors were dissected and fixed with $4 \%$ formaldehyde. Immunohistochemical analysis and HE staining were performed to pathologically analyze the tumor tissues complying with the standardized pathologic procedures and instructions (19). Sections were observed under the microscope by two pathologists with at least 5-year experience independently.

\section{Statistical analyses}

S-N-K method (one-way ANOVA) was utilized to process the comparison between groups after validating the assumption of normality. Kaplan-Meier ( $\log$ rank and Breslow) methods were used to compute the survival analyses. Statistical Package for the Social Sciences (SPSS) program (version 23.0; IBM, Armonk, USA) was used to process the data. The criterion for significance was set as $\mathrm{P}<0.05$.

\section{Results}

\section{In vitro experiments}

In the in vitro experiments, the cell proliferation assay, migration assay and real-time PCR were performed to assess the impact of ATO combined with VNP20009 on McA-RH7777 cells. The cell proliferation assay showed that the viable cells of ATO plus VNP20009 group were evidently less than those of other groups $(\mathrm{P}<0.001)$. The viable cells of both ATO group and VNP20009 group were less than that of the control group $(\mathrm{P}<0.05)$, but there was 


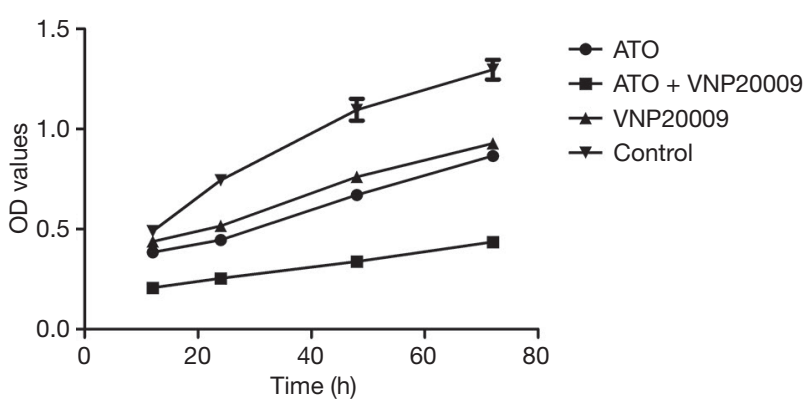

Figure 1 ATO combined with VNP20009 suppressed the proliferation of McA-RH7777 cells. CCK8 kit was used to assess the cell proliferation after the administration of ATO and/or VNP20009. The combination of ATO and VNP20009 had the strongest effect in terms of suppressing the proliferation of McARH7777 cells.

no significant difference between them (Figure 1).

In the migration assay, the cells that passed through the permeable membrane in ATO plus VNP20009 group were obviously less than those of other groups $(\mathrm{P}<0.05)$ (Figure 2), which suggested that ATO combined with VNP20009 could impair the migration ability of McA-RH7777 cells.

Real-time PCR analyses revealed that both VEGF and Vimentin levels in ATO plus VNP20009 group and VNP20009 group were lower than those of other groups $(\mathrm{P}<0.05)$ (Figure 3). VEGF is a key factor in the process of angiogenesis, which serves to promote tumor growth and metastasis. Vimentin is not only a diagnostic marker but also a hematogenous metastasis predictor for various carcinomas. It was suggested that ATO combined with VNP20009 could weaken the metastatic ability of McARH7777 cells.

\section{Primary tumor growth suppression and metastases inbibition}

To evaluate tumor responses to the joint therapy, all rats underwent MRI examinations on the 7th day and 14th day after the administration of ATO and VNP20009, respectively. MR images showed that the average viable tumor volume of ATO plus VNP20009 group was obviously smaller than those of other groups (Table 1, $\mathrm{P}<0.001$; Figure 4). Since lungs are the most common sites for HCC metastases, the micro-CT scans of chest were representatively performed to assess the metastases. The CT scans on the 14th day after the procedure revealed that the ratios of rats with lung metastases to the total rats in the ATO plus VNP20009 group and VNP20009 group were significantly lower than those of other groups $(0 \%$, $60 \%, 20 \%$, and $80 \%$ for ATO plus VNP20009 group, ATO group, VNP20009 group and control group, respectively; $\mathrm{P}<0.001$; Figure 5). Besides, there were no findings of other metastases on the imagines of MR of the 7th day and 14th day after the procedure.

\section{Survival analysis}

The median survivals of animals in each group were as follows: ATO plus VNP20009 group 75.0 \pm 4.38 days, ATO

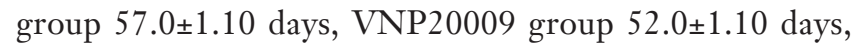
and control group 30.0 \pm 2.19 days, respectively. The KaplanMeier analyses demonstrated that the overall survival of ATO plus VNP20009 group was obviously longer than those of other groups $(\mathrm{P}<0.001$; Figure 6).

\section{HE staining and immunohistochemical analyses}

The tumor tissues were presented for HE staining and immunohistochemical analyses. HE staining showed that the tumor cells of ATO plus VNP20009 group were obviously less than those of other groups $(\mathrm{P}<0.001$; Figure 7). Immunohistochemical analyses displayed that Vimentin and VEGF levels in the ATO plus VNP20009 group were distinctly lower than those of other groups $(\mathrm{P}<0.001$; Figure 8).

\section{Discussion}

In this study, the strategy of ATO combined with VNP20009 in treating the advanced HCC was investigated. In the in vitro experiments, the results of proliferation assays and migration assays demonstrated that the combination of ATO and VNP20009 produced better effects of suppressing the cell proliferation and impairing the migration ability of McA-RH7777 cells than either ATO or VNP20009 alone. The real-time PCR analyses revealed that ATO combined with VNP20009 could effectively downregulate the expression of VEGF and Vimentin. Both VEGF and Vimentin play important roles in the process of tumor metastases. So it was suggested that the combination therapy might exert an inhibitory influence on the tumor metastases.

In the in vivo experiments, ATO mainly functioned in the well-oxygenated area of tumor to induce apoptosis and cell death, which could in turn create a favorable environment 

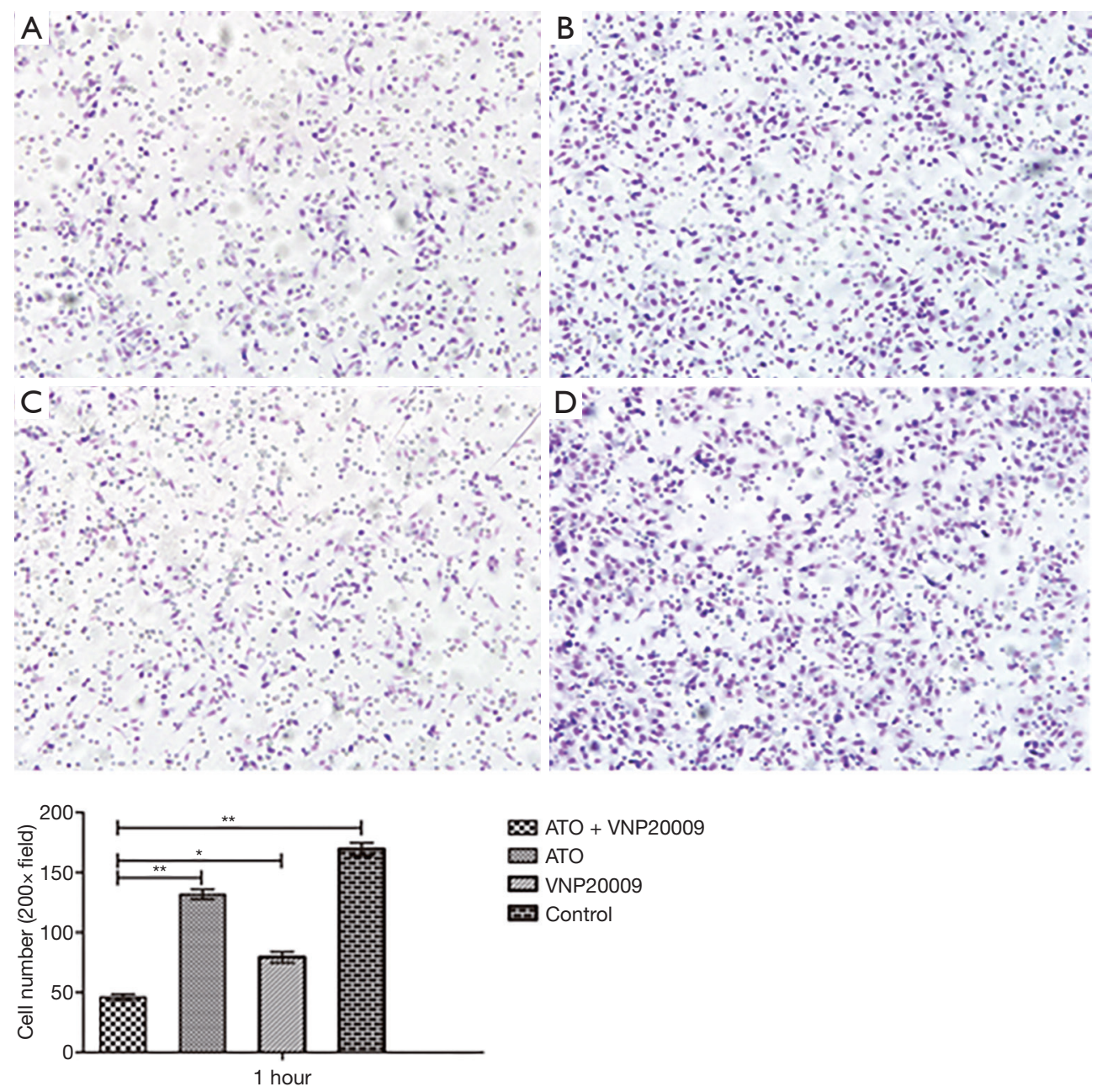

Figure 2 ATO combined with VNP20009 impaired the migration ability of McA-RH7777 cells. This experiment included four groups: (A) ATO plus VNP20009; (B) ATO; (C) VNP 20009; (D) Control. Transwell chambers were used to evaluate the migration ability in the experiment. The assay lasted for 12 hours. ATO combined with VNP20009 produced better effect in terms of impairing the migration ability of McA-RH7777 cells than either ATO or VNP20009 alone. * $\mathrm{P}<0.05 ;{ }^{* *}, \mathrm{P}<0.01$, magnification $\times 200$. Crystal violet staining was used.

for anaerobia, while VNP20009 had the advantage of colonizing the anoxic area of tumor and exerting anticancer properties. Thus, the combination of ATO and VNP20009 may produce a synergistic effect in treating the advanced HCC. The possible mechanisms of ATO in treating cancer include: down-regulation of genes BCL2; up-regulation of genes BAX, P16 and NM23; induction of tumor cell apoptosis; inhibition of cell proliferation, telomerase activity, and vascular endothelial growth factor; cytotoxic effects; and so on (20-22).

The mechanisms underlying the function of VNP20009 are very complex, which remain incompletely understood. In addition to direct toxicity to cancer cells, VNP20009 may exert an impact on the immune system and tumor microenvironment, which thus can be considered as a kind of immunotherapy. Up to date, the findings point to the increased immunogenicity of tumors caused by the accumulation of bacteria within tumor tissues and the alterations of innate immune cells modulated by the bacterial therapy (11).

The strategy of anaerobic bacterium combined with other modalities has been explored in several researches in literature. Avogadri et al. demonstrated that Salmonella in combination with low-dose radiotherapy could dampen tumor immune escape mechanisms and control the growth of distal established melanoma in a $\mathrm{CD}^{+} \mathrm{T}$ cell-dependent 


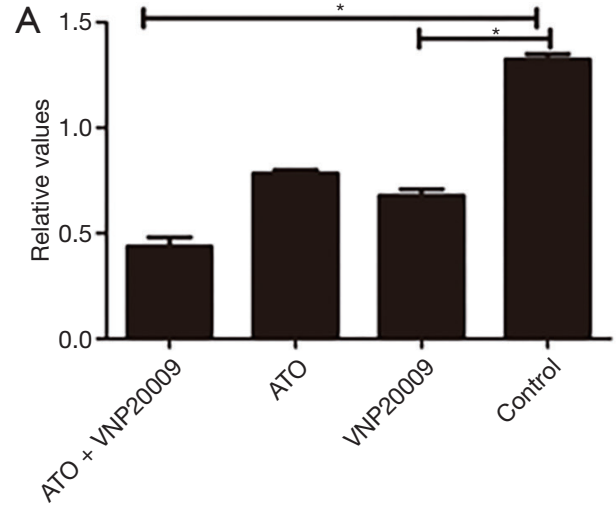

Vimentin

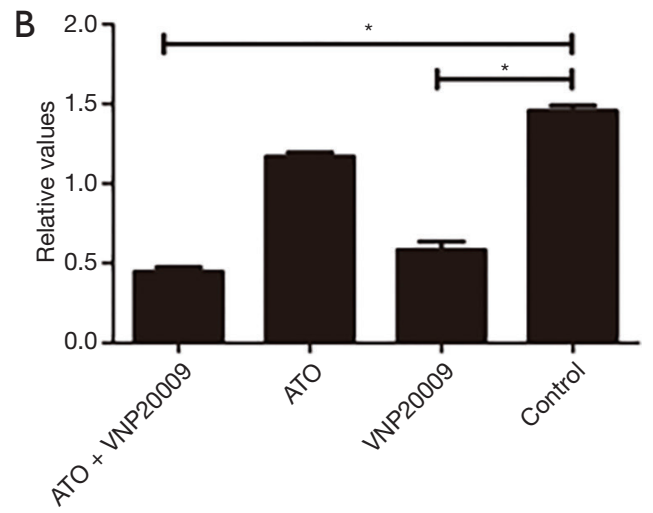

VEGF

Figure 3 ATO combined with VNP20009 decreased the expression of Vimentin and VEGF in McA-RH7777 cells. (A,B) Real-time PCR analyses displayed that both Vimentin and VEGF levels were the lowest in the ATO plus VNP20009 groups. Vimentin and VEGF were important markers related to the metastases. So, it was suggested that the joint strategy of ATO and VNP20009 might inhibit the metastases by downregulating the expression of Vimentin and VEGF. *, $\mathrm{P}<0.05$.

Table 1 Mean tumor volume of each group on the 7th day and 14th day measured by MRI

\begin{tabular}{lcc}
\hline \multirow{2}{*}{ Group } & \multicolumn{2}{c}{ Tumor volume, $\mathrm{mm}^{3}$} \\
\cline { 2 - 3 } & $7 \mathrm{~d}$ & $14 \mathrm{~d}$ \\
\hline ATO plus VNP20009 & $31.44 \pm 3.12$ & $124.21 \pm 25.76$ \\
ATO & $128.65 \pm 8.43$ & $3,011.43 \pm 421.23$ \\
VNP20009 & $78.53 \pm 7.52$ & $223.69 \pm 41.72$ \\
Control & $200.91 \pm 32.45$ & $4,186.85 \pm 427.74$ \\
\hline
\end{tabular}

The average tumor volume of ATO plus VNP20009 group was obviously smaller than those of other groups, $\mathrm{P}<0.001$.

manner (23). Dang et al. intravenously injected C. novyiNT spores together with conventional chemotherapeutic drugs such as dolastatin-10, mitomycin C, vinorelbine and docetaxel, producing extensive tumor necrosis and obvious anti-tumor effects (24). In our study, the results of MRI examinations also demonstrated that the combination of ATO and VNP20009 was more efficient than either VNP20009 or ATO alone in terms of suppressing tumor growth, which implied that a synergistic effect might be produced by the combination regime. The results of HE staining and immunohistochemical analyses were in accordance with this point.

Another encouraging observation made in our study was that the micro-CT scans of chest revealed that VNP20009 was capable of decreasing the rate of lung metastases. The possible mechanisms were related to the interaction between the bacteria and immune system. Chang et al. reported that Salmonella was able to convert an immunosuppressive tumor microenvironment into an immunogenic one by reducing the expression of immunosuppressive molecules (25). While tumor microenvironment has been proved to play an important role in the process of metastasis (26).

Based on the analyses of MRI and micro-CT, VNP20009 seemed to be more potent than ATO against HCC. However, it was noted that the median survival of animals appeared to be almost similar between the VNP20009 and ATO groups. There are several factors that may influence the survival of rats in the study. For example, as the number of rats in each group is relatively small, any accident deaths may exert an impact on the statistical result. Besides, one of the possible mechanisms of VNP20009 in treating tumors is suppressing tumor growth and metastasis by activating the systemic immune response. But excessive immune response can be harmful to the host if not be managed timely and properly, which may be an important factor influencing the survival of animals. It is necessary to further investigate this problem in the future.

The most common potential side effects of ATO are gastrointestinal damage and peripheral nerve impairment, such as nausea, vomit, impairing the liver function and renal function. In our study, no obvious decrease in physical activity and eating \& drinking ability was observed in the rats of ATO group. The dose of ATO in this study was chosen according to the instructions of clinically available ATO products. It is a pity that the dose range of ATO for 

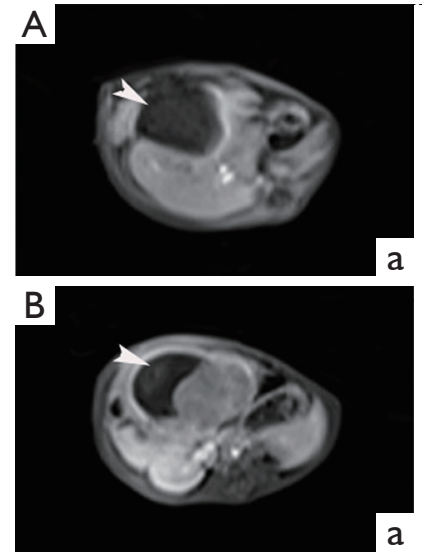
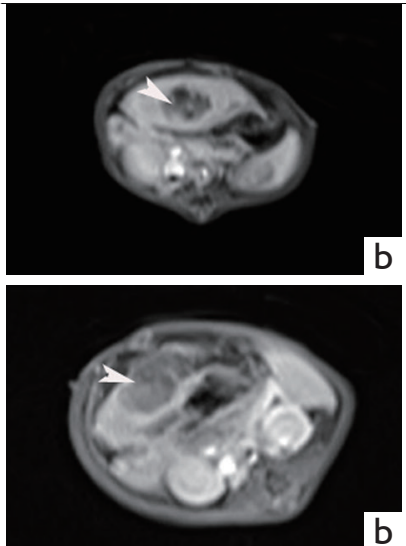
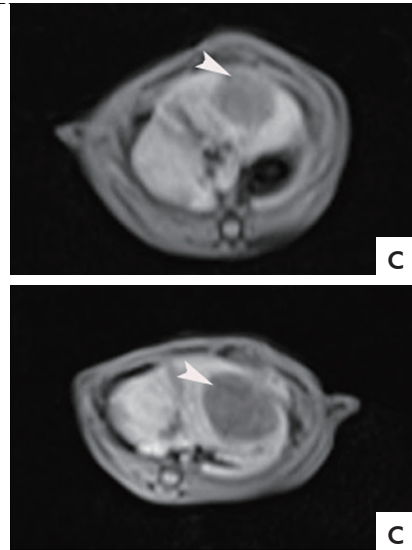
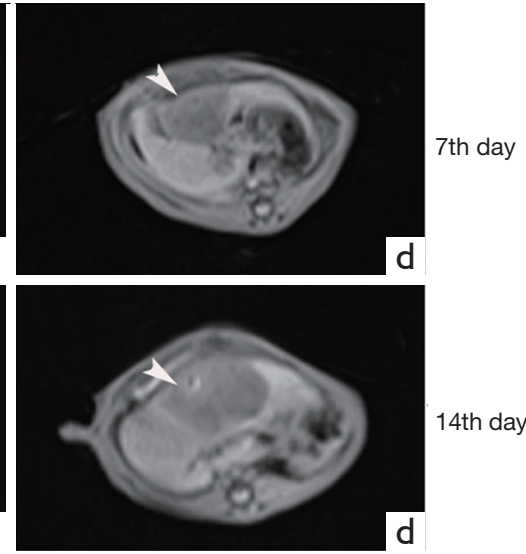

Figure 4 ATO combined with VNP20009 inhibited tumor growth in rat models. To evaluate tumor responses to the joint therapy, all rats underwent MRI examinations on the 7th day and 14th day after the administration of ATO and VNP20009, respectively. The arrows pointed to the tumors in the livers of the rats. The viable tumor tissues were enhanced in the tumors. (Aa,Ba) ATO plus VNP20009; (Ab,Bb) ATO; (Ac,Bc) VNP20009; (Ad,Bd) Control. The mean viable tumor volume of ATO plus VNP20009 group was distinctly smaller than those of other groups. $\mathrm{P}<0.001$.
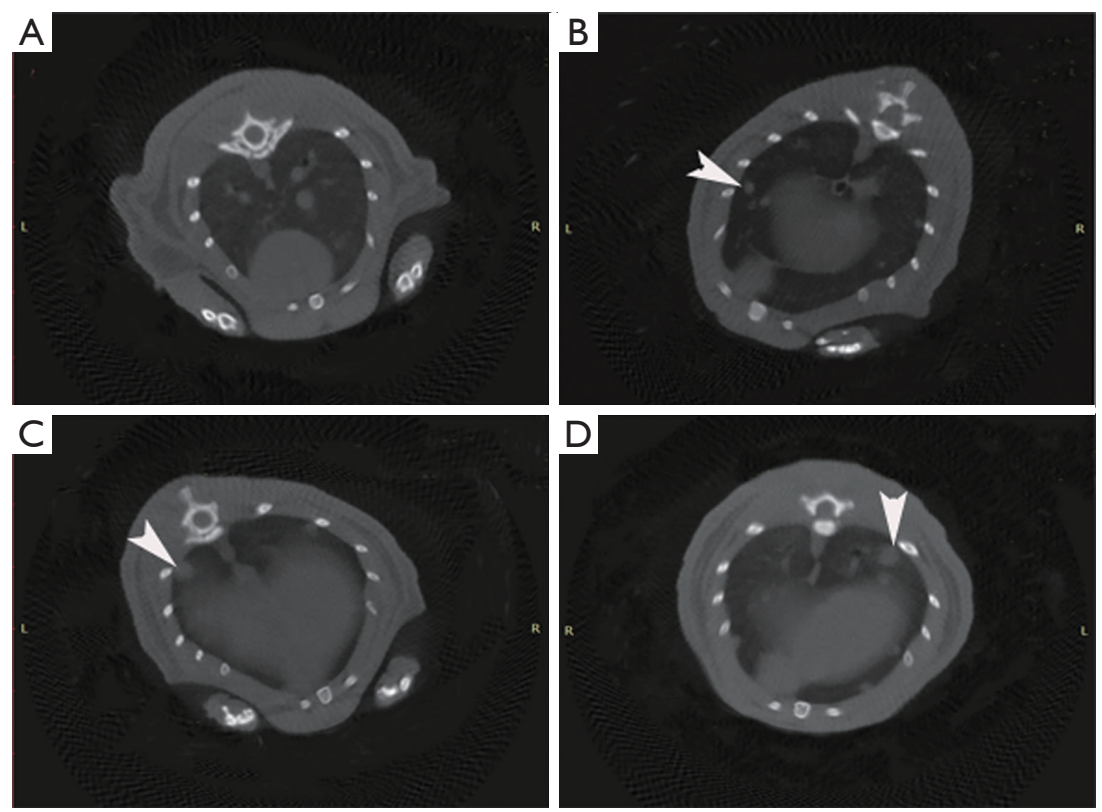

Figure 5 Micro CT scans of chest were representatively performed to assess the metastases. The CT scans on the 14th day after the procedure revealed that the ratios of rats with lung metastases to the total rats in the ATO plus VNP20009 group and VNP20009 group were significantly lower than those of other groups (0\%, 60\%, 20\%, and 80\% for ATO plus VNP20009 group, ATO group, VNP20009 group and control group, respectively; $\mathrm{P}<0.001$ ). The arrows pointed to the lesions of lung metastases in the rats. (A) ATO plus VNP20009; (B) ATO; (C) VNP20009; (D) control.

rats is not investigated in our study, which warrants further exploration in future study.

There are some limitations in our work. First, the number of subjects is relatively small. Second, the mechanisms of this strategy are not fully investigated. Further researches on the mechanisms involving such as 


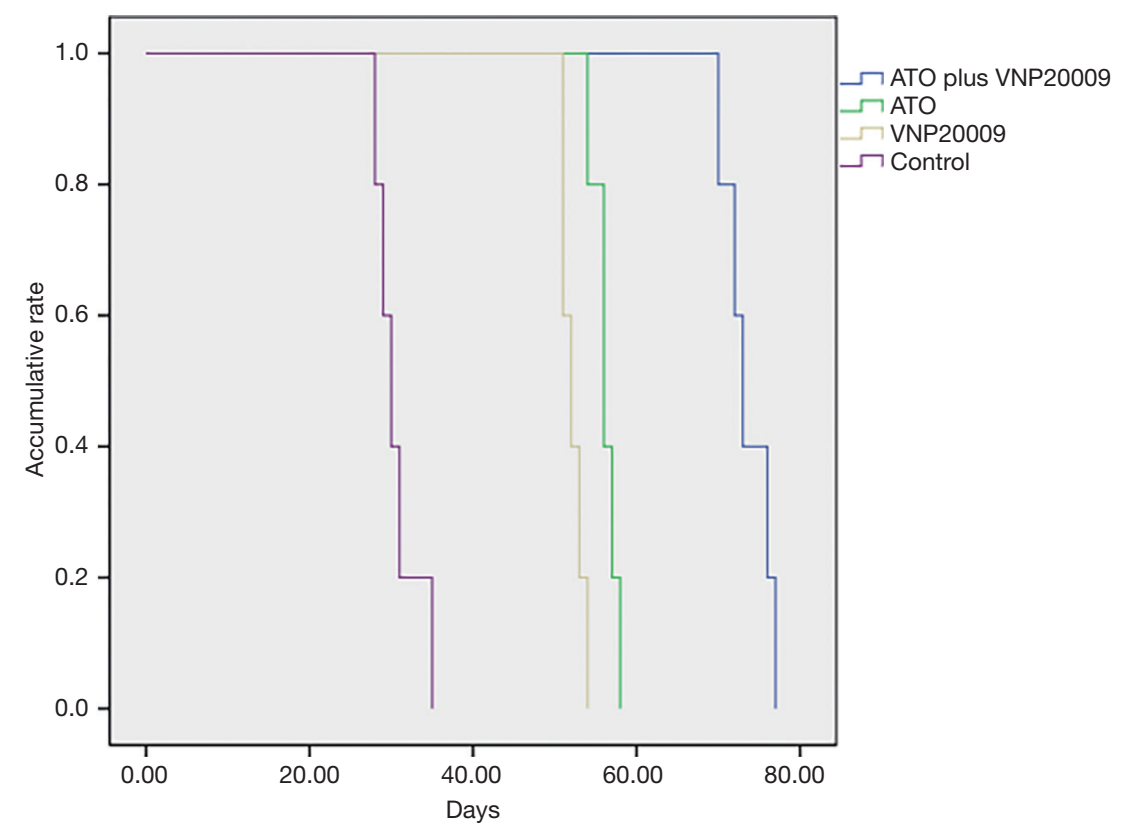

Figure 6 ATO combined with VNP20009 might prolong the overall survival of animal models. The Kaplan-Meier analyses demonstrated that the overall survival of ATO plus VNP20009 group was obviously longer than those of other groups. $\mathrm{P}<0.001$.
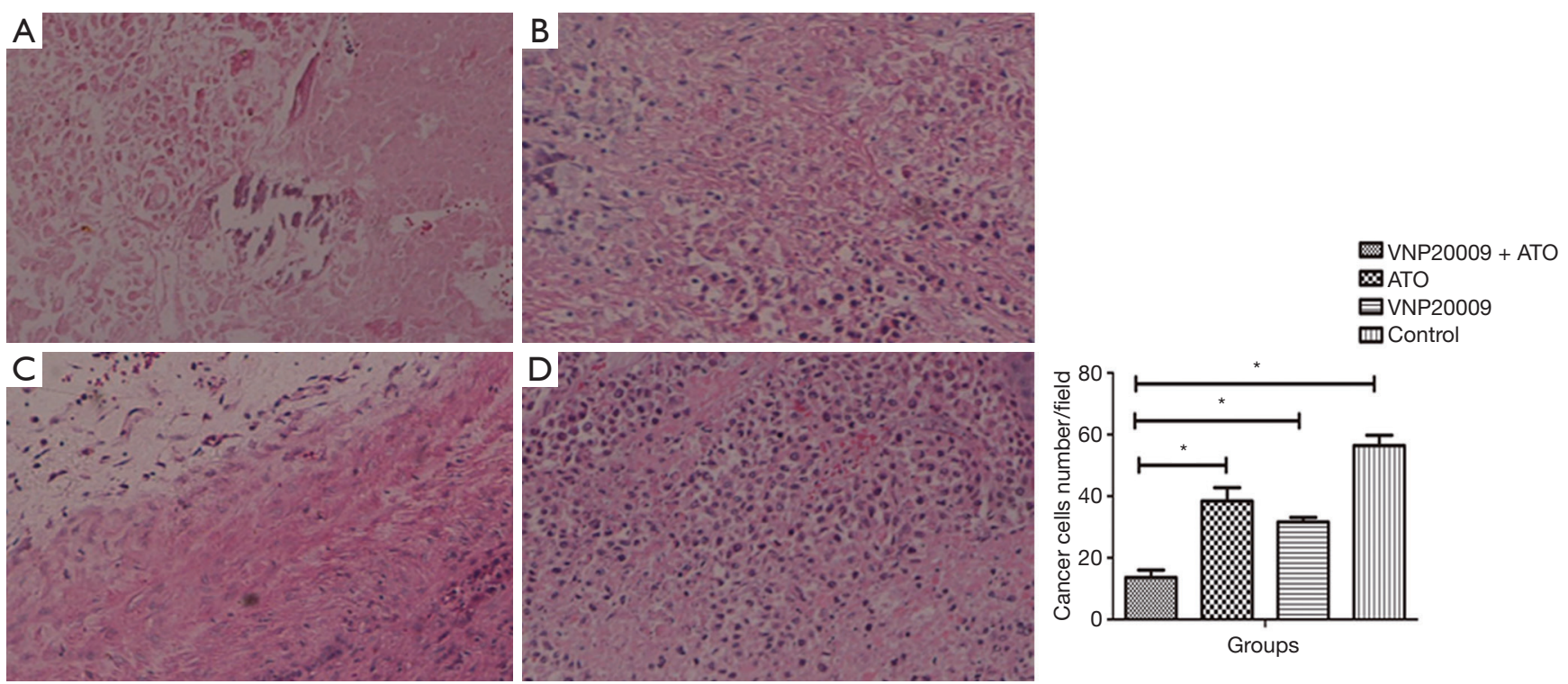

Figure 7 HE staining for tumor tissues. (A) ATO plus VNP20009; (B) ATO; (C) VNP20009; (D) Control. HE staining showed that the tumor cells of ATO plus VNP20009 group were obviously less than those of other groups, which were in accordance with the results of MRI analyses. *, $\mathrm{P}<0.001$; magnification $\times 10$.

immune system and apoptosis are required in the future. Third, in the context of joint therapy, further experiments to optimize the doses of agents and the intervals of each treatment are warranted. Last, the volume and weight of tumors were not measured after the rats were sacrificed in the study. It would be more impressive to display the gross 


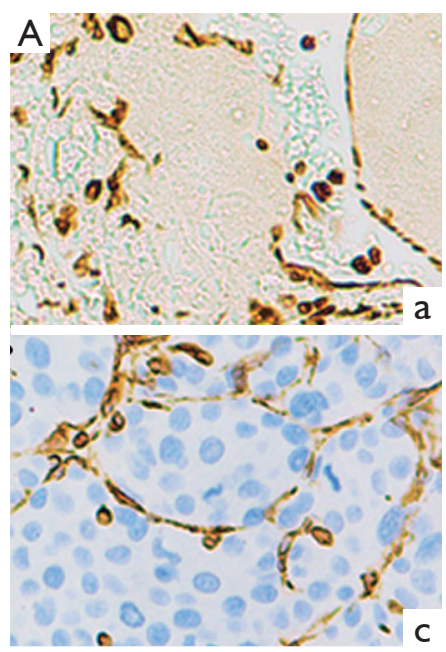

Vimentin

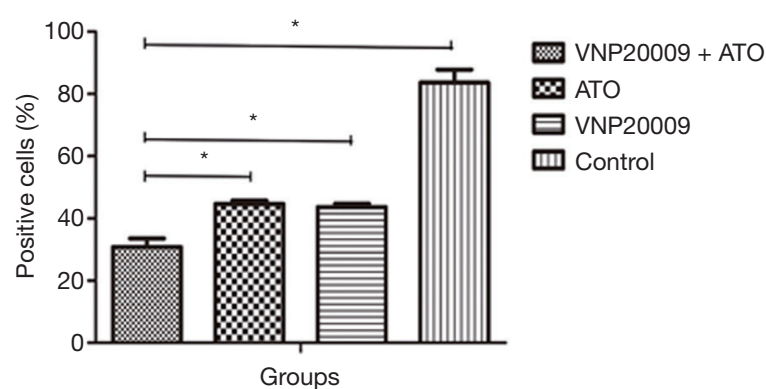

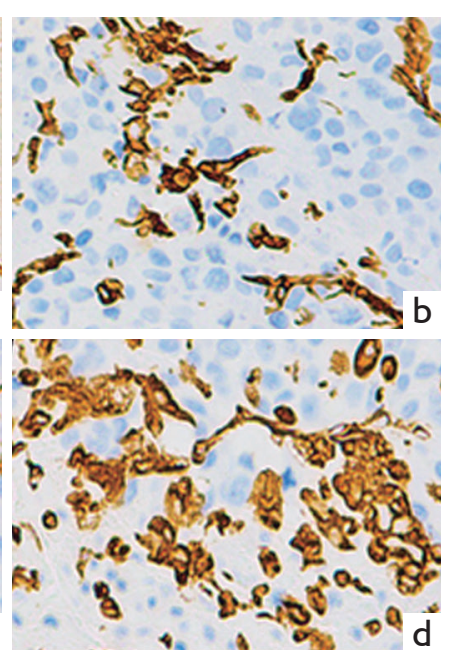

$\mathrm{d}$
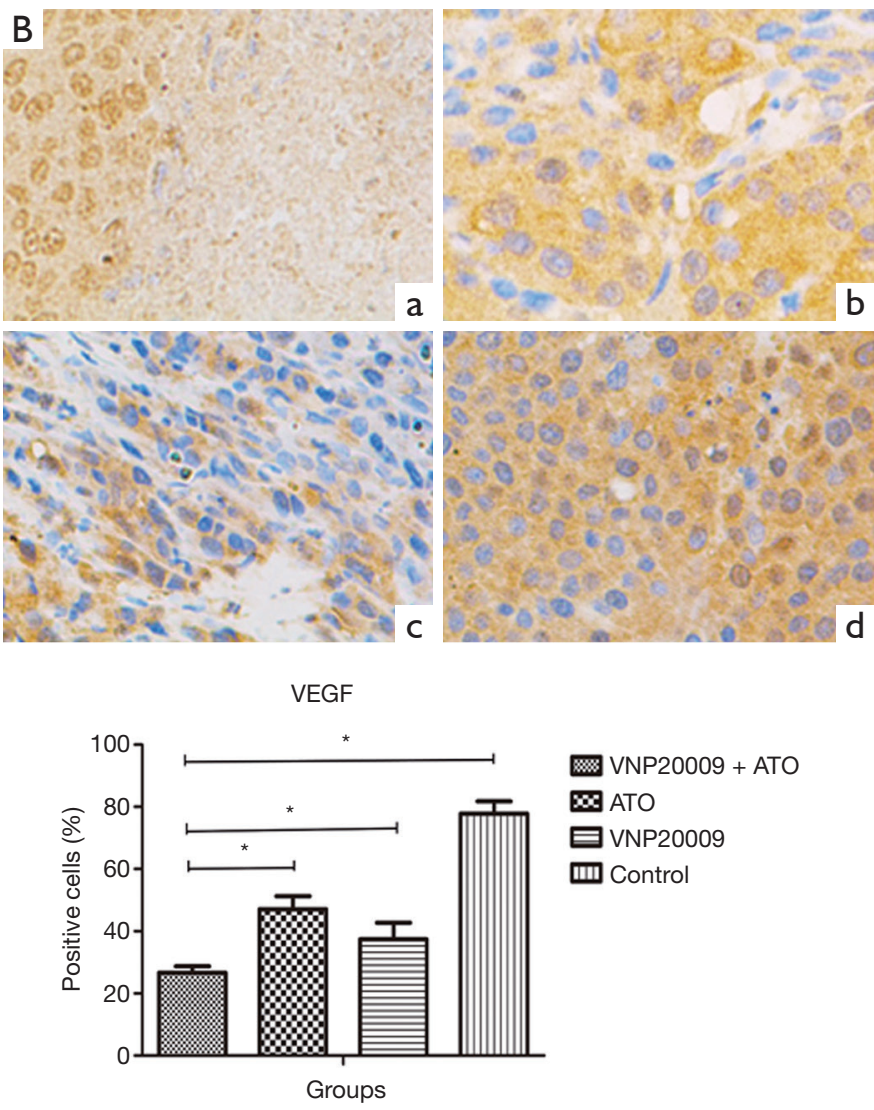

Figure 8 Immunohistochemical analyses for tumor tissues. (Aa,Ba) ATO plus VNP20009; (Ab,Bb) ATO; (Ac,Bc) VNP20009; (Ad,Bd) Control. The levels of VEGF and Vimentin in the tumors of ATO plus VNP20009 group were significantly lower than those of other groups, which were in compliance with the results of real-time PCR analyses. ${ }^{*}, \mathrm{P}<0.001$; magnification $\times 30$.

appearance of tumor tissues if the volume and weight of tumors were recorded.

\section{Conclusions}

In summary, this was an exploratory research and preliminary study to evaluate the synergistic effects of ATO combined with VNP20009 in treating the advanced HCC in rat models. This joint strategy is safe and effective and the two complementary interventions may have important synergistic effect. Further researches on the mechanisms of Salmonella typhimurium in treating cancer are warranted in the future.

\section{Acknowledgments}

We thank ELIXIGEN for the medical English editing service.

\section{Funding: None.}

\section{Footnote}

Reporting Checklist: The authors have completed the ARRIVE reporting checklist. Available at https://dx.doi. org/10.21037/jgo-21-4

Data Sharing Statement: Available at https://dx.doi. org/10.21037/jgo-21-4

Conflicts of Interest: All authors have completed the ICMJE uniform disclosure form (available at https://dx.doi. org/10.21037/jgo-21-4). The authors have no conflicts of interest to declare.

Ethical Statement: The authors are accountable for all aspects of the work in ensuring that questions related 
to the accuracy or integrity of any part of the work are appropriately investigated and resolved. Experiments were performed under a project license (NO. FUSCCIACUC-S20190337) granted by institutional ethics board of Fudan University Shanghai Cancer Centre, in compliance with Fudan University guidelines for the care and use of animals.

Open Access Statement: This is an Open Access article distributed in accordance with the Creative Commons Attribution-NonCommercial-NoDerivs 4.0 International License (CC BY-NC-ND 4.0), which permits the noncommercial replication and distribution of the article with the strict proviso that no changes or edits are made and the original work is properly cited (including links to both the formal publication through the relevant DOI and the license). See: https://creativecommons.org/licenses/by-nc-nd/4.0/.

\section{References}

1. Villanueva A. Hepatocellular Carcinoma. N Engl J Med 2019;380:1450-62.

2. Zhang P. On arsenic trioxide in the clinical treatment of acute promyelocytic leukemia. Leuk Res Rep 2017;7:29-32.

3. Liu B, Huang JW, Li Y, et al. Arsenic trioxide transarterial chemoembolization with and without additional intravenous administration of arsenic trioxide in unresectable hepatocellular carcinoma with lung metastasis: a single-blind, randomized trial. J Cancer Res Clin Oncol 2015;141:1103-8.

4. Wang $H$, Liu $Y$, Wang $X$, et al. Randomized clinical control study of locoregional therapy combined with arsenic trioxide for the treatment of hepatocellular carcinoma. Cancer 2015;121:2917-25.

5. Liu Y, Gao F, Song W. Periostin contributes to arsenic trioxide resistance in hepatocellular carcinoma cells under hypoxia. Biomed Pharmacother 2017;88:342-8.

6. Subramaniam M, In LL, Kumar A, et al. Cytotoxic and apoptotic effects of heat killed Mycobacterium indicus pranii (MIP) on various human cancer cell lines. Sci Rep 2016;6:19833.

7. Richardson MA, Ramirez T, Russell NC, et al. Coley toxins immunotherapy: a retrospective review. Altern Ther Health Med 1999;5:42-7.

8. Forbes NS. Engineering the perfect (bacterial) cancer therapy. Nat Rev Cancer 2010;10:785-94.

9. Liang K, Liu Q, Li P, et al. Genetically engineered Salmonella Typhimurium: Recent advances in cancer therapy. Cancer Lett 2019;448:168-81.

10. Igarashi K, Kawaguchi K, Kiyuna T, et al. Tumortargeting Salmonella typhimurium A1-R combined with recombinant methioninase and cisplatinum eradicates an osteosarcoma cisplatinum-resistant lung metastasis in a patient-derived orthotopic xenograft (PDOX) mouse model: decoy, trap and kill chemotherapy moves toward the clinic. Cell Cycle 2018;17:801-9.

11. Kaimala S, Al-Sbiei A, Cabral-Marques O, et al. Attenuated Bacteria as Immunotherapeutic Tools for Cancer Treatment. Front Oncol 2018;8:136.

12. Toso JF, Gill VJ, Hwu P, et al. Phase I study of the intravenous administration of attenuated Salmonella typhimurium to patients with metastatic melanoma. J Clin Oncol 2002;20:142-52.

13. Zhang XB, Wang P, Zhou DY. Imaging characteristics of orthotopic rat hepatocellular carcinoma model induced by transplantation of McA-RH7777 cells. Zhonghua Gan Zang Bing Za Zhi 2009;17:59-61.

14. Low KB, Ittensohn M, Luo X, et al. Construction of VNP20009: a novel, genetically stable antibioticsensitive strain of tumor-targeting Salmonella for parenteral administration in humans. Methods Mol Med 2004;90:47-60.

15. Wang GZ, Fang ZT, Zhang W, et al. Increased metastatic potential of residual carcinoma after transarterial embolization in rat with McA-RH7777 hepatoma. Oncol Rep 2014;31:95-102.

16. Lee YJ, Lee JM, Lee JS, et al. Hepatocellular carcinoma: diagnostic performance of multidetector CT and MR imaging-a systematic review and meta-analysis. Radiology 2015;275:97-109.

17. Méndez O, Zavadil J, Esencay M, et al. Knock down of HIF-1alpha in glioma cells reduces migration in vitro and invasion in vivo and impairs their ability to form tumor spheres. Mol Cancer 2010;9:133.

18. Laurent F, Montaudon M, Corneloup O. CT and MRI of Lung Cancer. Respiration 2006;73:133-42.

19. Chen CS, Zhao Q, Qian S, et al. Ultrasound-guided RNA interference targeting HIF-1 alpha improves the effects of transarterial chemoembolization in rat liver tumors. Onco Targets Ther 2015;8:3539-48.

20. Maeda H, Hori S, Nishitoh H, et al. Tumor growth inhibition by arsenic trioxide (As2O3) in the orthotopic metastasis model of androgen-independent prostate cancer. Cancer Res 2001;61:5432-40.

21. Moon Y, Park G, Kim Y, et al. Arsenic trioxide (As2O3) sensitivity of carcinoma cell lines and cancer cells from 
patients with carcinomatosis peritonei. Ann Clin Lab Sci 2004;34:271-6.

22. Yu J, Qian H, Li Y, et al. Therapeutic effect of arsenic trioxide (As2O3) on cervical cancer in vitro and in vivo through apoptosis induction. Cancer Biol Ther 2007;6:580-6.

23. Avogadri F, Mittal D, Saccheri F, et al. Intra-tumoral Salmonella typhimurium induces a systemic anti-tumor immune response that is directed by low-dose radiation to treat distal disease. Eur J Immunol 2008;38:1937-47.

Cite this article as: Zhao Q, Wang Y, Li WT. Synergistic effects of arsenic trioxide combined with Salmonella typhimurium in treating the advanced hepatocellular carcinoma in rat models. J Gastrointest Oncol 2021;12(4):1732-1742. doi: 10.21037/jgo21-4
24. Dang LH, Bettegowda C, Huso DL, et al. Combination bacteriolytic therapy for the treatment of experimental tumors. Proc Natl Acad Sci U S A 2001;98:15155-60.

25. Chang SY, Kim YJ, Ko HJ. Potential therapeutic antitumor effect of a Salmonella-based vaccine. Hum Vaccin Immunother 2013;9:1654-60.

26. Yang L, Lin PC. Mechanisms that drive inflammatory tumor microenvironment, tumor heterogeneity, and metastatic progression. Semin Cancer Biol 2017;47:185-95. 